\title{
Chapter 7. CKD and CVD: cardiorenal association
}

(C) Japanese Society of Nephrology 2009

- CKD increases the morbidity and mortality rate of myocardial infarction, heart failure, and stroke.

- CKD and CVD share many of risk factors in common.

- In a case of CVD, it is necessary to confirm whether CKD underlies CVD.

- A CKD patient is more likely to die possibly from CVD than from ESKD. Figure 7-1 shows a comparison of CKD patients who died prior to transplant/dialysis and those who progressed to ESKD in the general population in the US according to the levels of kidney function. Even among patients with CKD stage 4 (GFR 15-29) die from CVD at a far higher rate than they progress to ESKD. Furthermore, patients with proteinuria died from CVD more often than those without proteinuria. This is also the case with CKD patients in advanced stages $3-4$.

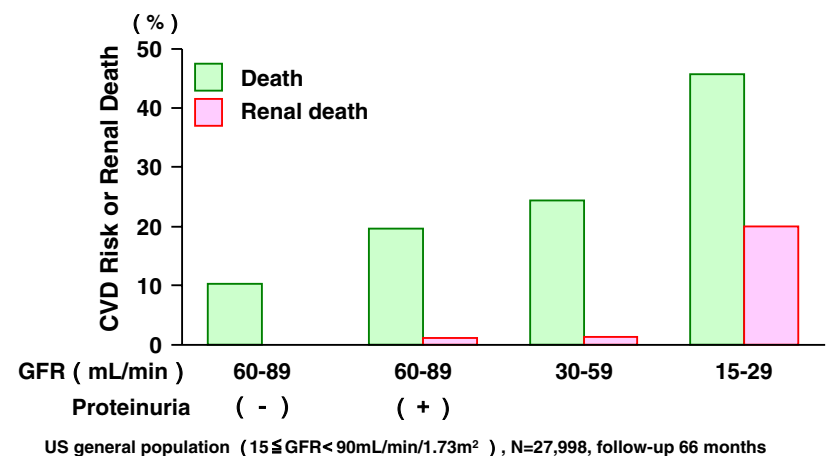

Fig. 7-1 Comparison of the rate of death prior to transplant/dialysis and that of renal replacement therapy. Data are quoted, with modification, from Keith DS et al. [Arch Intern Med 2004;164(6): 659-663]
- It has been reported not only in Europe and the US, but also in Japan that mildly reduced kidney function or proteinuria is the great risk factor for myocardial infarction and stroke. It is strongly suggested that CKD patients in Japan may have more chance of dying from CVD than of surviving until ESKD. It is necessary to examine for the presence of CVD in CKD patients.

- However, it has been reported that CVD patients tend to have reduced kidney function (Fig. 7-2). In patients who had suffered myocardial infarction, one-third of the patients had reduced kidney function as bad as CKD stage 3 or greater. Furthermore, a risk of recurrent infarction increased in advanced stages of CKD during a 3-year follow-up period after initial attack (Fig. 7-3). CKD, therefore, is a major risk factor for CVD.

- Figure 7-4 illustrates common risk factors shared by both CKD and CVD grouped by the impairment of fluid regulation and endothelium damage. Being in either of

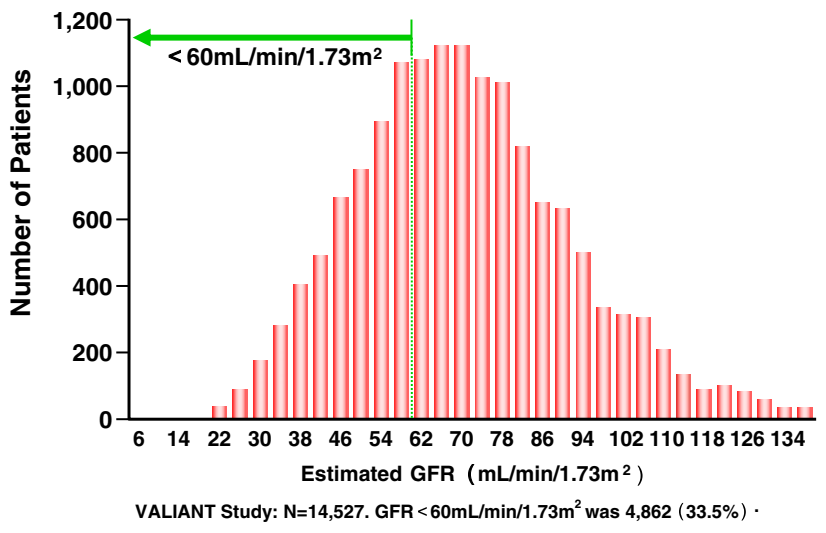

Fig. 7-2 Distribution of estimated GFR at baseline among 14,527 patients. Data are quoted, with modification, from Anavekar NS et al. [N Engl J Med 2004;351(13):1285-1295] 
Fig. 7-3 Kaplan-Meier estimates of the rates of death at 3 years from cardiovascular $(C V)$, causes reinfarction, congestive heart failure $(\mathrm{CHF})$, stroke, resuscitation after cardiac arrest, and the composite end point, according to the estimated GFR at baseline. Data are quoted, with modification, from Anavekar NS et al. [N Engl J Med 2004;351(13):1285-1295]

Fig. 7-4 Cardiorenal association through anemia, volume dysregulation, endothelial damage, and atherosclerosis

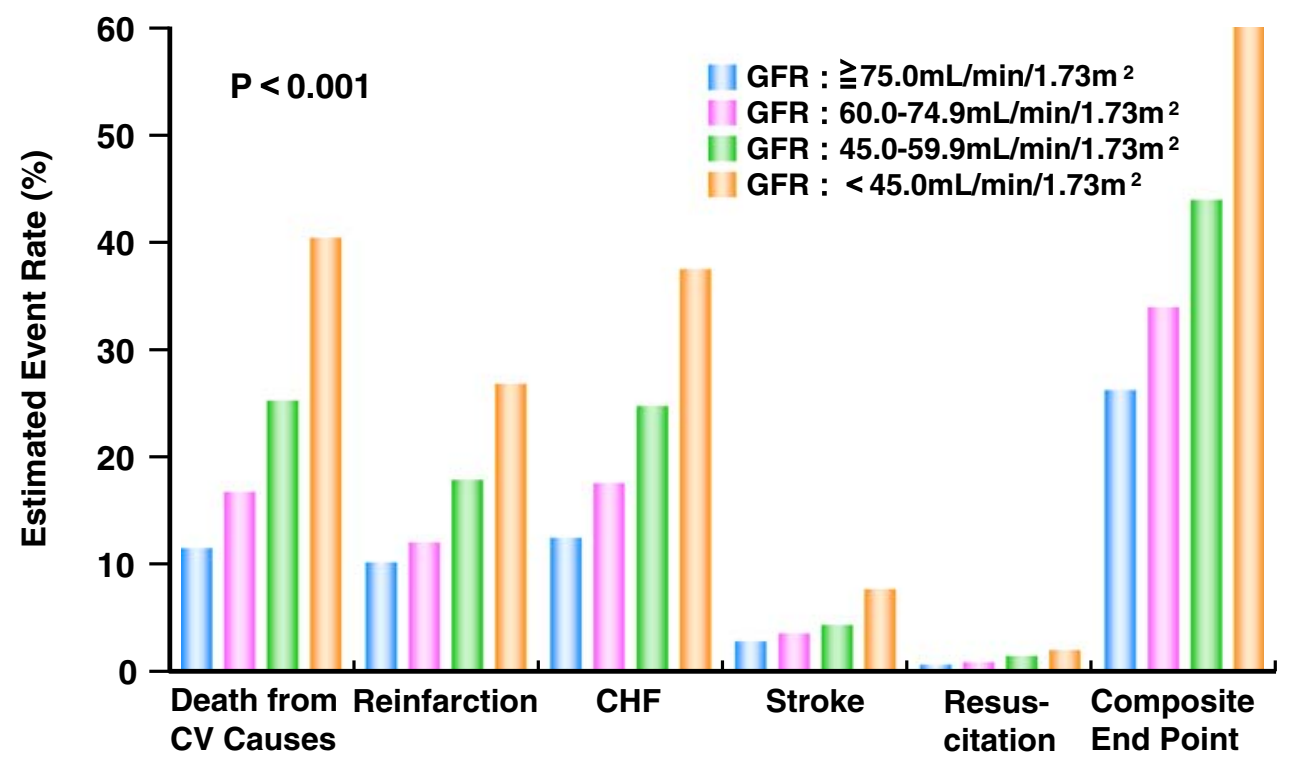

VALIANT Study: acute myocardial infarction 14,527

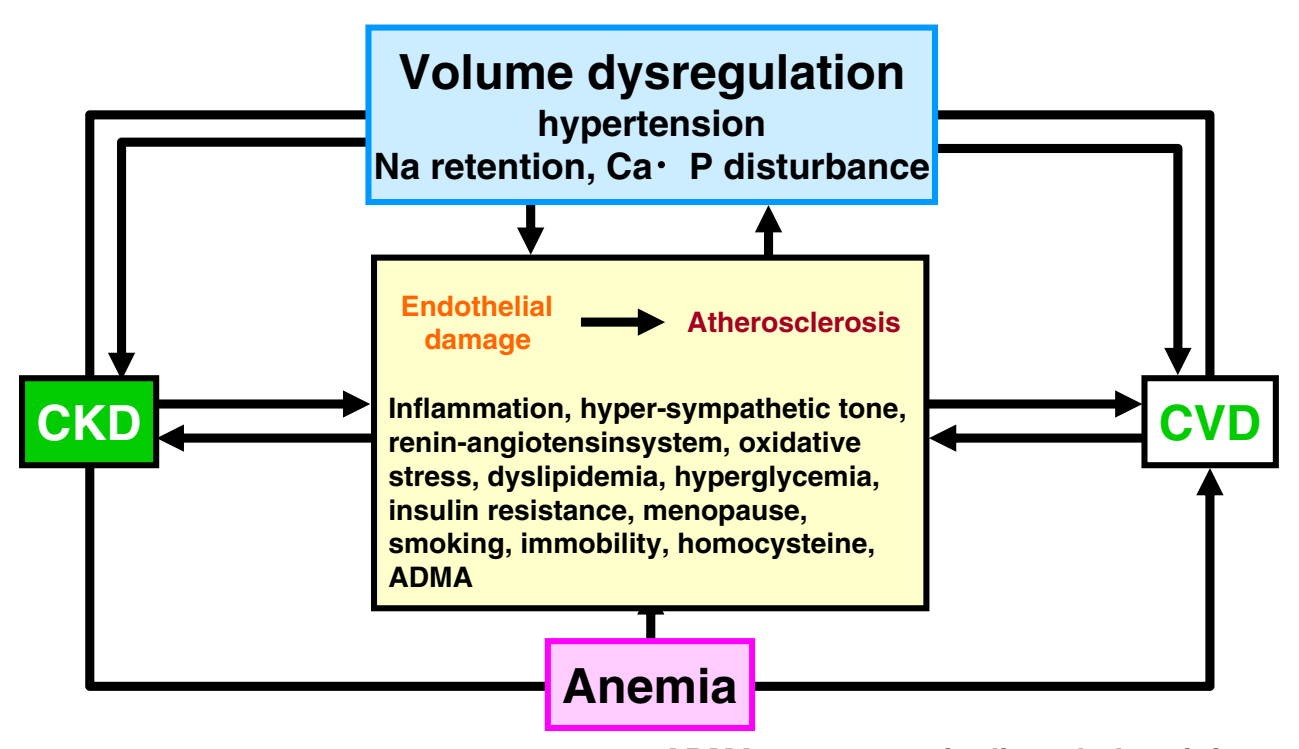

ADMA : asymmetric di-methyl arginine these two groups can accelerate atherosclerosis and cause cardiovascular burden generated by hypervolemia. Renal anemia, one of comorbidities of CKD, is also an independent risk factor for CVD. It is important that risk factors should be treated at best to prevent the development and progression of CVD as well as aggravation of CKD. 